\title{
Inquérito farmacoepidemiológico de pacientes de meia idade e idosos em três comunidades de Cascavel, PR - Brasil. Verificação evolutiva do conhecimento terapêutico
}

\author{
Jorge Juarez Vieira Teixeira*1, Fabiola Giordani Cano ${ }^{1}$, Andréia Cristina Conegero Sanches ${ }^{1}$, \\ Tatiane Aparecida Carniel', Deborah Sandra Leal Guimarães Schneider ${ }^{2}$ \\ ${ }^{1}$ Centro de Ciências Médicas e Farmacêuticas, Universidade Estadual do Oeste do Paraná, \\ ${ }^{2}$ Centro de Ciências Exatas e Tecnológicas, Universidade Estadual do Oeste do Paraná
}

*Correspondência:

J. J. V. Teixeira

Centro de Ciências Médicas e

Farmacêuticas

Universidade Estadual do Oeste do Paraná

Rua Universitária, 1619

85819-110 - Cascavel - Paraná,Brasil

E-mail: jorgetei@usp.br;

jorgetei@hotmail.com
O objetivo da pesquisa foi identificar variáveis relacionadas ao conhecimento da terapêutica nos periodos pré e pós-intervenção e características sócio-demográficas de 80 pacientes de meia idade e idosos (45 anos ou mais) em três comunidades paroquiais de Cascavel-PR. Desenvolveu-se um inquérito farmacoepidemiológico, seguido de estudo prospectivo no periodo de novembro de 2003 a dezembro de 2004, e os dados foram coletados em formulário semiestruturado. Houve predominância para o sexo feminino (82,5\%), idade média de 67,3 anos, escolaridade de menos de oito anos de estudo (75,0\%), maioria casado (57,5\%), morando apenas o casal (63,7\%), renda de 1 a 3 salários (80\%), número médio de medicamentos prescritos na fase pré $(4,3)$ e na pós-intervenção $(3,5)$. A classe de fármacos mais prescrita foi para o sistema cardiovascular $(48,2 \%)$. As variáveis, leitura da bula e freqüencia, conhecimento sobre o medicamento, esquecimento da dose prescrita, satisfação com o medicamento e importância das informações, embora apresentassem diferenças na freqüencia relativa do periodo pré-intervenção para o pós-intervenção, não sustentaram diferença estatisticamente significativa. Apesar desse resultado, é preciso considerar que a diferença quanto ao grau de evolução dos resultados apresentam relevância clinica, pendendo favoravelmente a relação beneficio/risco.

\author{
Unitermos \\ - Conhecimento terapêutico/ \\ avaliação \\ - Farmacoepidemiologia \\ - Inquérito \\ farmacoepidemiológico
}

\section{INTRODUÇÃO}

Os idosos pertencem ao grupo etário que mais consome medicamento, quando comparado com outras faixas etárias, utilizando de 30 a $35 \%$ dos fármacos prescritos
(Salom, Davies 1995; Piraino, 1995). No Brasil, pouco se conhece sobre a farmacoepidemiologia dos idosos, estudos de base populacional ainda são escassos (Loyola Filho et al., 2006). Para Nóbrega et al. (2005), deve-se atentar para o fato de que o organismo do idoso apresenta mudanças em 
suas funções fisiológicas que não devem ser desconsideradas, podendo levar a uma farmacocinética diferenciada e maior sensibilidade tanto aos efeitos terapêuticos quanto adversos dos fármacos.

A cadeia farmacoterapêutica pela sua alta complexidade exige atualização continuada dos profissionais de saúde, principalmente se o objetivo da terapia é a manutenção de bom nível da adesão medicamentosa. Revisão bibliográfica sobre a adesão à prescrição medicamentosa realizada por Trostle (1988) mostra que mais de 4000 pesquisas em língua Inglesa e artigos de revisão a respeito do tema estão listados no Index Medicus e em outras obras de referências bibliográficas. Das mais de 4000 pesquisas investigadas em âmbito internacional, já se dispõe de um número superior a 250 variáveis como possíveis etiologias do não seguimento da medicação (Haynes, 1979). Aproximadamente metade dos pacientes em uso contínuo de medicamentos não adere ao tratamento estabelecido pelo médico. Nos EUA a não adesão responde por 40 a $55 \%$ das prescrições. Esta situação é grave porque expõe o paciente a um maior risco de hospitalização e morbidade (Morrow et al., 1988; Kessler, 1992).

Paralelamente a adesão, as reações adversas são responsáveis por parcela de 10 a 31\% das admissões agudas em geriatria (Nolan, O’Malley 1988; Lamy, 1990; Atkin, Shenfield, 1995). Aos problemas negativos propiciados pela farmacoterapia, cabe aos profissionais da área da saúde tentar amenizá-los. Nesse sentido, a comunicação e a informação dos fármacos prescritos aos pacientes poderiam garantir resultados mais satisfatórios da terapia farmacológica. Informar a população é uma das formas de minimizar os riscos referentes às intoxicações e ocorrências de eventos adversos (Tierling et al., 2004). A obtenção de sucesso em um tratamento medicamentoso está relacionada com o nível de informação que o paciente possui acerca do medicamento (Pires Júnior, Mengue, 2005).

Inúmeros fatores contribuem para o conhecimento reduzido do paciente idoso relacionado ao tratamento medicamentoso. Dentre as várias causas, destacam-se a falta de aconselhamento individualizado, a falta de informação escrita personalizada e reforço das instruções orais, a inabilidade para recordar as informações previamente apresentadas e a ausência de um ajudante ou auxiliar na hora de tomar a medicação (O’Connell, Johnson, 1992). Aliada a estes fatos, fica limitada a compreensão das informações contidas nas bulas dos medicamentos, em grande parte pela própria condição de não-conhecimento técnico, tornando as bulas em sua maioria insatisfatórias quanto aos itens "Informações ao Paciente" e "Informações técnicas" (Gonçalves et al., 2002). Muitas vezes, o bulário deixa o idoso desnecessariamente preocupado com os efeitos adversos mencionados, pois as informações sobre a gravidade e freqüência destes efeitos são insuficientes (Silva et al., 2000).

A comunicação com os pacientes pode ser útil não somente para ajudá-los a entender como utilizar adequadamente o medicamento, mas também para fornecer ao paciente a motivação necessária para o tratamento (Schommer et al., 2002). A informação e o conhecimento com a finalidade de capacitar os pacientes sobre a terapia prescrita e a doença, se destacam como um instrumento motivador poderoso para melhoria da qualidade de vida e da resposta necessária para a farmacoterapia ótima.

Tierling et al. (2004) indicam a necessidade de atuação dos profissionais da área da saúde na qualificação do conhecimento da população usuária de medicamentos. Prover educação continuada aos usuários de medicamentos passa a ser tarefa inadiável destes profissionais.

A pesquisa teve como objetivo identificar variáveis relacionadas ao conhecimento da terapêutica nos períodos pré-intervenção e pós-intervenção e características sóciodemográficas de pacientes de meia idade e idosos no espaço comunitário.

\section{MATERIAL E MÉTODOS}

A pesquisa desenvolvida utilizou-se de diferentes métodos, sendo inicialmente, inquérito farmacacoepidemiológico pré-intervenção, seguido de estudo prospectivo em três comunidades paroquiais de Cascavel (Jardim Universitário, Jardim Maria Luiza e Jardim Itália). Após, realizou-se o inquérito pós-intervenção. O período para o desenvolvimento da investigação foi de novembro de 2003 a dezembro de 2004. As paróquias foram escolhidas, considerando a distribuição periférica, intermediaria e central.

O primeiro momento da pesquisa ocupou-se de uma ampla divulgação nas paróquias envolvidas, seguido de seis palestras técnicas: hipertensão arterial, diabetes mellitus, pneumonias, conhecendo o medicamento que toma, atividade física e alimentação saudável. Aos pacientes interessados, houve a apresentação do objetivo do estudo, da importância para saúde pública e do termo de consentimento livre e esclarecido, aprovado pelo Comitê de Ética em Pesquisa da Universidade Estadual do Oeste do Paraná - Cascavel/Paraná - Brasil, protocolo 295/2003.

O critério para inclusão dos pacientes foi: possuir diagnóstico médico de no mínimo uma doença crônica nãotransmissível, utilizar ao menos um medicamento de uso contínuo por no mínimo um ano e faixa etária de 45 anos e mais. Aos concordantes foi aplicado primeiramente um formulário estruturado previamente testado em população semelhante de Cascavel para as informações sócio- 
demográficas (idade, sexo, escolaridade, estado civil e renda). A seguir foi empregado o formulário da pré-intervenção para conhecer o nível de informação das variáveis farmacoepidemiológicas (leitura da bula e freqüência, esquecimento da dose prescrita, satisfação com o medicamento e importância das informações recebidas pelo paciente sobre a terapêutica). O nível de informação foi identificado de acordo com as afirmações relatadas pelo paciente. Ao término da pesquisa o mesmo instrumento de coleta de dados foi reaplicado (pós-intervenção) para comparação dos dados.

Os participantes foram acompanhados individualmente após as seis palestras e orientados por oito meses consecutivos por pesquisadores treinados (acadêmicos de farmácia, enfermagem e medicina). As informações sobre a função do fármaco prescrito, valorização da concentração prescrita, horário e período do tratamento recomendado, conservação, importância da adesão ao tratamento, interações medicamentosas e efeitos adversos eram fornecidas aos pacientes periodicamente. $\mathrm{O}$ contato foi realizado mensalmente por meio de visita ou telefone, dependendo da necessidade ou da disponibilidade da comunicação, pois nem todos dispunham de telefone na residência.

Inicialmente a amostra foi constituída de 100 pacientes de meia idade e idosos. Houve perda de 20 candidatos por diversos motivos (mudança de endereço, recusa em continuar a participar, não utilizar mais medicamento, endereço errado e não seguimento farmacoterapêutico por oito meses). A amostra foi aleatorizada pelo comparecimento do entrevistado na paróquia do dia 06 de agosto a 24 de setembro de 2003 e constituiu-se de 80 pacientes do sexo masculino e feminino. Os dados foram tabulados e analisados com auxílio do programa Epi Info versão windows 2000 e Bioestat 3.0. Para a análise estatística utilizou-se o teste dos sinais, correspondendo as hipóteses $\mathrm{H}_{\mathrm{o}}:=0 ; \mathrm{H}_{1}:>0$

\section{RESULTADOS E DISCUSSÃO}

As variáveis sócio-demográficas analisadas destacam idade média de 67,3 anos (DP=8,73) com variação de 47 a 87 anos e maioria $(81,2 \%)$ com 60 anos ou mais, sexo feminino (82,5\%), estado civil casado $(57,5 \%)$, menos de oito anos de escolaridade $(75,0 \%)$, morando apenas o casal $(63,7 \%)$ e renda de 1 a 3 salários mínimos para $80,0 \%$ dos pacientes (Tabela I). Loyola Filho et al. (2006), em estudo epidemiológico de base populacional com 1598 idosos da região metropolitana de Belo Horizonte, relataram que a média de idade dos entrevistados foi de 69,7 anos. Investigação desenvolvida em Porto Alegre com 141 idosos mostrou que $66 \%$ dos entrevistados eram mulheres, sendo que para $53 \%$ os casais moravam juntos e $67 \%$ possuíam 8 anos de escolaridade (Flores, Mengue, 2005). Os dois estudos apresentam semelhanças com a pesquisa desenvolvida, embora a faixa etária apresente cortes diferenciados.

O número médio de medicamentos prescritos aos pacientes na fase pré-intervenção foi de 4,3 $(\mathrm{DP}=2,5)$ com extensão de 1 a 14, sendo que $43,8 \%$ utilizam de 1 a 3, 37,5\% de 4 a 6 e $18,7 \%$ de 7 a 14 medicamentos. Por outro lado, na fase pós-intervenção foi de 3,5 (DP=1,9), variando de 1 a 12 medicamentos e $62,8 \%$ dos pacientes utilizam de 1 a 3 medicamentos, $32,1 \%$ de 4 a 6 , e $5,1 \%$ de 7 a 12 . Investigação com pacientes de meia idade e idosos da periferia de Brasília, apresentou número médio de medicamentos de 3,2 ( $\mathrm{DP}=2)$ (Nóbrega et al., 2005). Em outro estudo, apesar dos pacientes idosos serem institucionalizados, houve concordância com o estudo ora desenvolvido, onde o número médio de medicamentos foi de 3,03 $(\mathrm{DP}=1,99)$ por paciente, e $72,4 \%$ utilizavam entre 1 a 4 (Correr et al., 2007). Dentre os 357 fármacos, os mais prescritos foram para o aparelho cardiovascular $(48,2 \%)$, com maior evidência para o captopril (38/172), pertencente a classe dos inibidores da enzima conversora de angiotensina, seguidos pelo sistema nervoso central $(19,6 \%)$, tendo a dipirona $(26 / 70)$ como representante e o aparelho digestivo e metabolismo $(10,1 \%)$, com destaque para a glibenclamida (9/36)) (Tabela II). Os fármacos do aparelho cardiovascular também foram destaque nos estudos desenvolvidos por Flores e Mengue (2005) na cidade de Porto Alegre com 215 idosos, ocupando o primeiro lugar (32,0\%), seguido pelo sistema nervoso central $(22,0 \%)$ e por Coelho Filho et al. (2004) com 668 idosos na cidade de Fortaleza, com 29,3\% para o cardiovascular e $13,5 \%$ para o nervoso central.

As variáveis de conhecimento da terapêutica chamam a atenção para situações próximas e diferenciadas (Tabela III). Em relação à leitura da bula do medicamento na fase pré-intervenção $83,7 \%$ afirmaram ser importante, porém, $36,3 \%$ sempre a lêem. Já na fase pós, $87,4 \%$ relataram ser importante a sua leitura, onde a freqüência para categoria sempre foi de $38,7 \%$. As farmácias devem prover o paciente de informações qualificadas e de simples entendimento. Gonçalves et al (2002), relatam que geralmente as farmácias não dispõem de profissional habilitado para orientar o consumidor. A bula poderia servir como fonte confiável e adequada de informação, suprindo em parte essa carência; entretanto não é capaz de fazê-lo satisfatoriamente, por não cumprir inteiramente seu papel como instrumento informativo.

Na etapa pré-intervenção da variável conhecimento sobre o medicamento, houve destaque para as categorias bom $(52,4 \%)$, contra $42,4 \%$ na $2^{\text {a }}$ fase, aparentando redução de freqüência, porém as categorias regular e bom foram deslocadas para a categoria ótimo. Silva et al. (2000) relatam 
TABELA I - Variáveis sócio-demográficas de pacientes de meia idade e idosos de três comunidades de Cascavel - Paraná, novembro 2003 a dezembro de 2004.

\begin{tabular}{|c|c|c|c|}
\hline Variável & Categoria & $\mathrm{N}(80)$ & $(\%)$ \\
\hline \multirow[t]{2}{*}{ Sexo } & Masculino & 14 & 17,5 \\
\hline & Feminino & 66 & 82,5 \\
\hline \multirow[t]{4}{*}{ Estado civil } & Casado & 46 & 57,5 \\
\hline & Solteiro & 1 & 1,2 \\
\hline & Viúvo & 27 & 33,8 \\
\hline & Desquitado/divorciado** & 6 & 7,5 \\
\hline \multirow[t]{5}{*}{ Escolaridade } & Não lê/não escreve & 9 & 11,3 \\
\hline & Sabe ler*** & 4 & 5,0 \\
\hline & Menos de 8 anos & 60 & 75,0 \\
\hline & De 8 a 11 anos & 6 & 7,5 \\
\hline & Acima de 11 anos & 1 & 1,2 \\
\hline \multirow[t]{3}{*}{ Renda } & Menos que $1 \mathrm{SM}^{*}$ & 9 & 11,3 \\
\hline & 1 a 3 & 64 & 80,0 \\
\hline & 4 a 6 & 7 & 8,7 \\
\hline \multirow[t]{5}{*}{ Com quem mora } & Sozinho & 11 & 13,8 \\
\hline & $1^{\mathrm{a}}$ geração (esposo/a) & 51 & 63,7 \\
\hline & $2^{\mathrm{a}}$ geração (esposa/a + filho + genro $)$ & 11 & 13,8 \\
\hline & $3^{\mathrm{a}}$ geração (esposa/a + fillho/genro + neto) & 6 & 7,5 \\
\hline & Amigo & 1 & 1,2 \\
\hline
\end{tabular}

*SM (Salário mínimo médio do período do estudo=111US\$), ** Legalmente separados, *** Não teve participação na educação formal (Aprendeu com parentes)

TABELA II - Fármacos prescritos a pacientes de meia idade e idosos, segundo ATC, Cascavel - Paraná, novembro de 2003 a dezembro de 2004.

\begin{tabular}{lccc}
\hline Classificação Anátomo Terapêutico Química/ATC & Código ATC & $\mathrm{N}$ & $(\%)$ \\
\hline Aparelho cardiovascular & $\mathrm{C}$ & 172 & 48,2 \\
Sistema nervoso central & $\mathrm{N}$ & 70 & 19,6 \\
Aparelho digestivo e metabolismo & $\mathrm{A}$ & 36 & 10,1 \\
Aparelho musculoesquelético & $\mathrm{M}$ & 22 & 6,2 \\
Órgãos dos sentidos & $\mathrm{S}$ & 14 & 3,9 \\
Aparelho geniturinário e hormônios sexuais & $\mathrm{G}$ & 13 & 3,6 \\
Aparelho respiratório & $\mathrm{R}$ & 06 & 1,7 \\
Hormônios de uso sistêmico, excetos sexuais. & $\mathrm{H}$ & 03 & 0,8 \\
Outros & $\mathrm{V}$ & 21 & 5,9 \\
Total & & 357 & 100,0 \\
\hline
\end{tabular}

que ao avaliar o nível de informação a respeito de medicamentos prescritos a pacientes ambulatoriais de um hospital universitário, por volta de um terço dos pacientes possuía nível de informação bom. Visitas domiciliares realizadas por farmacêuticos a pacientes de alto risco com 45 anos e mais nos EUA, não provocaram mudança estatisticamente significativa entre o grupo estudado e controle sobre o conhecimento e práticas referentes aos medicamentos. Os farmacêuticos alegaram di- ficuldades para obtenção de mudanças de atitudes sobre os medicamentos na população estudada (Sidel et al.,1990). Pesquisa randomizada controlada relativa ao impacto da intervenção do farmacêutico clínico nos EUA, possibilitou o aumento da adesão e de maior conhecimento pelo paciente sobre o seu regime terapêutico (Lipton, Bird, 1994).

Referente ao esquecimento da dose prescrita do fármaco na $1^{\text {a }}$ fase, $31,2 \%$ esperam e tomam a próxima 
TABELA III - Variáveis farmacoepidemiológicas pré e pós-intervenção de pacientes de meia idade e idosos de três comunidades de Cascavel - Paraná, novembro de 2003 a dezembro de 2004.

\begin{tabular}{|c|c|c|c|c|}
\hline Pré - intervenção Variáveis & $(\%)$ & Pós - intervenção Categorias & $(\%)$ & Valor $\mathrm{p}$ \\
\hline \multirow[t]{3}{*}{ Leitura da bula } & 83,7 & Importante & 87,4 & 0,875 \\
\hline & 8,8 & Não importante & 6,3 & \\
\hline & 7,5 & Depende & 6,3 & \\
\hline \multirow[t]{5}{*}{ Freqüência da leitura da bula } & 36,3 & Sempre & 38,7 & 0,499 \\
\hline & 13,7 & Quase sempre & 17,5 & \\
\hline & 18,8 & Às vezes & 16,3 & \\
\hline & 13,7 & Raramente & 15,0 & \\
\hline & 17,5 & Nunca & 12,5 & \\
\hline \multirow[t]{5}{*}{ Conhecimento sobre o medicamento } & 20,0 & Ótimo & 45,0 & 0,937 \\
\hline & 52,4 & Bom & 42,4 & \\
\hline & 20,0 & Razoável & 6,3 & \\
\hline & 6,3 & Ruim & 5,0 & \\
\hline & 1,3 & Péssimo & 1,3 & \\
\hline \multirow[t]{7}{*}{ Esquecimento da dose prescrita } & 6,3 & Não esquece & 0,0 & 0,811 \\
\hline & 31,2 & Espera e toma a próxima dose & 27,5 & \\
\hline & 27,4 & Toma quando se lembra & 36,2 & \\
\hline & 6,3 & Toma somente no dia seguinte & 6,3 & \\
\hline & 2,5 & Toma as duas doses juntas & 0,0 & \\
\hline & 1,3 & Depende & 1,3 & \\
\hline & 25,0 & Outra & 28,7 & \\
\hline \multirow[t]{5}{*}{ Satisfação com o medicamento prescrito } & 20,0 & Muito satisfeito & 32,5 & 0,937 \\
\hline & 65,0 & Satisfeito & 63,8 & \\
\hline & 5,0 & Pouco satisfeito & 0,0 & \\
\hline & 3,7 & Nem satisfeito, nem insatisfeito & 3,7 & \\
\hline & 6,3 & Insatisfeito & 0,0 & \\
\hline \multirow[t]{4}{*}{ Importância das informaçõesrecebidas } & 67,5 & Sim & 97,4 & 0,937 \\
\hline & 22,5 & Não & 0,0 & \\
\hline & 5,0 & Depende & 1,3 & \\
\hline & 5,0 & Não sei & 1,3 & \\
\hline
\end{tabular}

dose e $27,4 \%$ tomam quando se lembram, enquanto que na fase pós $27,4 \%$ esperam e tomam a próxima dose e $36,2 \%$ tomam quando se lembram. Os valores encontrados em ambas as fases oferecem poucos elementos para análise, considerando que a migração foi mínima de uma categoria para outra, salvo engano, não produzindo impacto sobre os resultados da variável esquecimento da dose. Dewulf et al. (2006) ao investigar a adesão ao tratamento medicamentoso em 110 pacientes com doenças gastrintestinais crônicas em Ribeirão Preto, destacam que dentre os não aderentes $(58,2 \%)$, mais de dois terços apresentavam comportamento de baixa adesão do tipo não intencional, indicando padrões de esquecimento ou descuido com o horário para tomar o medicamento.

Em relação à terapêutica prescrita, inicialmente $85,0 \%$ estavam satisfeitos ou muito satisfeitos, e posteri- ormente esse índice aumentou para 96,3\%. Quanto à importância das informações recebidas pelo paciente sobre a farmacoterapia, $67,5 \%$ consideram-nas como importante, elevando-se ao final do estudo para 97,4\%. Dudas et al. (2001) chamam a atenção para o impacto do seguimento de 221 pacientes com idade média de 57 anos, via telefone após alta hospitalar. Número maior de pacientes do grupo intervenção que receberam as ligações telefônicas estavam mais satisfeitos com as instruções recebidas sobre os medicamentos do que os pertencentes ao grupo controle, que não receberam as ligações $(\mathrm{P}=0,007)$.

Apesar das variáveis pré e pós-intervenção mostrarem diferenças quanto às freqüências relativas iniciais $\mathrm{e}$ finais, não houve diferença estatisticamente significativa, mostrando forte evidência que a intervenção educativa no período do estudo, não foi suficiente para alterar nenhuma 
das variáveis estudadas. Por outro lado, essas diferenças apresentam relevância clinica, pendendo para uma relação benefício/risco favorável para os pacientes. Inúmeros estudos de intervenção farmacêutica têm mostrado resultados animadores; redução de custos, melhora das prescrições, maior promoção da adesão do paciente a terapêutica e controle da possibilidade de reações adversas (Romano-Lieber et al., 2002).

Algumas limitações devem ser valorizadas nesta investigação. Em se tratando de inquérito descritivo, prospectivo e principalmente com amostra de conveniência, é recomendado a não generalização dos dados, devido a não validade externa. Coelho Filho et al. (2004) destacam que todo inquérito domiciliar ao utilizar procedimento sistemático, pode estar sujeito a vícios por parte dos entrevistadores. Outra questão, diz respeito ao estudo prospectivo por oito meses, que pode ter criado necessidades e desejos de respostas positivas dos entrevistados junto ao pesquisador. A pesquisa embora apresente limitações, possui a sua importância como estudo preliminar, pois o número de publicações científicas sobre experiências bem ou mal sucedidas em relação a farmacoterapia do adulto e do idoso em ambiente comunitário ou hospitalar brasileiro é inexpressiva.

\section{CONCLUSÃO}

Os achados do estudo evidenciam população feminina, idosa, baixo nível socioeconômico, residindo o casal no domicílio, utilizando acima de três medicamentos prescritos, prevalecendo o sistema cardiovascular.

As variáveis farmacoepidemiológicas destacam crescimento em suas categorias de maior grau. Embora não estatisticamente significativo, é possível inferir que esses valores sobre o conhecimento do medicamento, da satisfação com o medicamento prescrito e da importância das informações fornecidas pelos pesquisadores, poderiam provocar aumento da adesão à terapêutica e da significância clínica.

\section{ABSTRACT \\ Pharmacoepidemiological survey of middle-age and elderly patients in three communities of Cascavel, PR - Brazil: Progressive verification of their therapeutic knowledge}

Variables on therapeutic knowledge in pre-and post-intervention periods and the social and demographic characteristics of 80 middle-aged and elderly (45 years or more) patients in three parish communities in Cascavel, PR Brazil are provided. A pharmacoepidemiological survey was developed; a prospective study between November 2003 and December 2004 was undertaken; data were collected in a semi-structured form. Females (82.5\%) were predominant; average age 67.3 years old; schooling less than 8 years; most of them married (57.5\%); only the couple inhabits the house (63.7\%); income 1 to 3 salaries (80\%); mean numbers of prescribed medicines pre-and post-intervention were 4.3 and 3.5, respectively. Medicine for the cardiovascular system (48.2\%) was the most described (48.2\%). Variables - reading information on drug and its frequency, knowledge on the medicine used and importance of information - were statistically non-significant, although difference in relative frequency from the pre- to the post-intervention period existed. Nevertheless, difference with regard to degree of progress in results shows clinical relevance with a favorable trend towards the benefit/risk ratio.

UNITERMS: Therapeutic knowledge. Pharmacoepidemiology. Pharmacoepidemiological survey.

\section{REFERÊNCIAS BIBLIOGRÁFICAS}

ATKIN, P.A.; SHENFIELD, G.M. Medication-related adverse reactions and the elderly: a literature review. $A d v$. Drug. React. Toxicol. Rev., v.14, n.3, p.175-91, 1995.

CORRER, C.J.; PONTAROLO, R.; FERREIRA L.C.; BAPTISTÃO, S.A.M. Riscos de problemas relacionados com medicamentos em pacientes de uma instituição geriátrica. Rev. Bras. Cienc. Farm., v.43, n.1, p.55-62, 2007.

COELHO FILHO, J.M.; MARCOPITO, L.F.; CASTELO A. Perfil de utilização de medicamentos por idosos em área urbana do nordeste do Brasil. Rev. Saúde Pública, v.38, n.4, p.557-64, 2004.

DEWULF, N.L.S.; MONTEIRO, R.A.; PASSOS, A.D.C.; VIEIRA, E.M.; TRONCON, L E. A. Adesão ao tratamento medicamentoso em pacientes com doenças gastrintestinais crônicas acompanhadas no ambulatório de um hospital universitário. Rev. Bras. Cienc. Farm., v.42, n.4, p.575-584, 2006.

DUDAS, V.; BOOKWALTER, T.; KERR, K. M.; PANTILAT, S. Z. The impact of follow-up telephone calls to patients after hospitalization. Am. J. Med., v.111, n.9B, p.26S-30S, 2001. 
FLORES, L.M.; MENGUE, S.S. Uso de medicamentos por idosos em região do sul do Brasil. Rev. Saúde Pública, v.39, n.6, p.924-929, 2005.

GONÇALVES, S.A.; MELOB, G.; TOKARSKI, M.H.L.; BARBOSA-BRANCO, A. Bulas de medicamentos como instrumento de informação técnico-científica. Rev. Saúde Pública, v.36, n.1, p.33-39, 2002.

HAYNES, R.B. Introduction. In: HAYNES, R. B.; SACKETT, D. L.; TAYLOR, D. W. (Eds.). Compliance in Health Care. Baltimore: Johns Hopkins University Press, 1979. p.1-7.

KESSLER, D.A.A. challenge for American pharmacists. Am. Pharm., v.NS32, n.1, p.33-36, 1992.

LAMY, P. Adverse drug effects. Clin. Geriatr. Med., v.6, n.2, p.293-307, 1990.

LIPTON, H.L., BIRD, J.A. The impact of clinical pharmacists' consultations on geriatric patients' compliance and medical care use: a randomized controlled trial. Gerontologist, v.34, n.3, p.307-315, 1994.

LOYOLA FILHO, A.I.; UCHOA, E.; LIMA-COSTA, M.F. Estudo epidemiológico de base populacional sobre uso de medicamentos entre idosos na região metropolitana de Belo Horizonte, Minas Gerais, Brasil. Cad. Saúde Pública, v.22, n.12, p.2657-2667, 2006.

MORROW, D.; LEIRER, V.; SHEIKH, J. Adherence and medication instructions: review and recommendations. $J$. Am. Geriatr. Soc., v.36, n.12, p.1147-1160, 1988.

NÓBREGA, O.T.; MELO, G.F.; KARNIKOWSKI, M.G.O. Pattern of drugs prescribed for community-residing middle-aged and older adults from the outskirts of Brasília. Rev. Bras. Cienc. Farm, v.41, n.2, p.271-277, 2005.

NOLAN, L.; O’MALLEY, K. Prescribing for the elderly: part II prescribing patterns: differences due to age. J. Am. Geriatr. Soc., v.36, n.3, p.245-254, 1988.

O'CONNELL, M.B.; JOHNSON, J.F. Evaluation of medication knowledge in elderly patients. Ann. Pharmacother., v.26, n.7, 8, p.919-921, 1992.

PIRAINO, A.J. Managing medication in the elderly. Hosp Practice, v.30, n.6, p.59-64, 1995.
PIRES JUNIOR, J. V.; MENGUE, S. S. Análise do nível de informação sobre medicamentos antimicrobianos por pacientes de um centro de saúde de Porto Alegre, Brasil. Acta Farm. Bonaerense, v.24, n 1, p.134-138, 2005.

ROMANO-LIEBER, N.S.; TEIXEIRA J.J.V.; GOULARTE FARHAT, F.C.L.; RIBEIRO, E.; CROZATTI, M.T.L.; OLIVEIRA, G.S.A. Revisão dos estudos de intervenção do farmacêutico no uso de medicamentos por pacientes idosos. Cad. Saúde Pública, v.18, n.6, p.1499-1507, 2002.

SALOM, I.L.; DAVIS, K. Prescribing for older patients: How to avoid toxic drug reactions. Geriatrics, v.50, n.10, p.3740, 43-45, 1995.

SCHOMMER, J.C.; BYERS, S.R.; PAPE, L. L.; CABLE, G. L.; WORLEY, M. M.; SHERRIN, T. Interdisciplinary medication education in a church environment. Am. J. Health Syst. Pharm., v.59, n.5, p.423-428, 2002.

SIDEL, V.W.; BEIZER, J.L.; LISI-FAZIO, D.; KLEINMANN, K.; WENSTON, J.; THOMAS, C.; KELMAN, H. R. Contolled study of the impact of educational home visits by pharmacists to high-risk older patients. J. Commun. Health, v.15, n.3, p.163-174, 1990.

SILVA T.; DAL-PIZZOL F.; BELLO, C.M, MENGUE, S.; SCHENKEL, E.P. Bulas de medicamentos e a informação adequada ao paciente. Rev Saúde Pública, v.34, n.2, p.184-189. 2000.

TIERLING, V. L.; PAULINO, M. A.; FERNANDES, L. C.; SCHENKEL, E. P.; MENGUE, S. S. Nível de conhecimento sobre a composição de analgésicos com ácido acetilsalicílico. Rev. Saúde Pública, v.38, n.2, p.223-227, 2004.

TROSTLE, J.A. Medical compliance as an ideology. Soc. Sci. Med., v.27, n.12, p.1299-1308, 1988.

Recebido para publicação em 24 de julho de 2007 Aceito para publicação em 05 de dezembro de 2007 